\title{
Simultaneous Laparoscopic Nephrectomy and Colectomy for Synchronous Primary Malignancies: Surgical Techniques and the Results of Two Cases
}

\author{
Campanati Luca $^{1}$, Coccolini Federico ${ }^{1, *}$, Lotti Marco ${ }^{1}$, Deho Federico ${ }^{2}$, Deiana Gianfranco ${ }^{2}$, \\ Lazzareschi Daniel $^{1}$, Poiasina Elia ${ }^{1}$, Pisano Michele ${ }^{1}$, Magnone Stefano ${ }^{1}$, Colaianni Nicola ${ }^{1}$, Poletti \\ Eugenio $^{1}$, Da Pozzo Luigi ${ }^{2}$, Ansaloni Luca ${ }^{1}$ \\ ${ }^{1}$ General Surgery dept. Papa Giovanni XXIII hospital, Bergamo, Italy \\ ${ }^{2}$ Urology dept., Papa Giovanni XXIII hospital, Bergamo, Italy \\ *Corresponding Author: federico.coccolini@gmail.com
}

Copyright (C) 2013 Horizon Research Publishing All rights reserved.

\begin{abstract}
A series of randomized studies have revealed comparable long-term results for both laparosopic and open resections in the treatment of renal and colonic neoplasms. From a purely oncological perspective, the benefit-detriment debate regarding synchronous laparoscopic resections remains a hotly disputed issue and continues to face scrutiny in today's medical community. However, although this debate has become an important issue, there are few reports in mainstream literature discussing the simultaneous laparoscopic resection of renal and colorectal carcinomas. This report overviews two entirely laparoscopic procedures: a simultaneously performed right nephrectomy and left colectomy and a simultaneously performed left nephrectomy and left colectomy. The laparoscopic technique for simultaneous abdominal primary malignancy has proven to be safe, effective, and practicable. This paper will overview the authors' experience with such procedures, outlining technical setbacks in the course of surgery and discussing the overall results of the operations.
\end{abstract}

Keywords Synchronous Resection, Laparoscopy, Nephrectomy, Colectomy, Technique, Educational

\section{Introduction}

Widespread use of CT scans for pre-operative staging in the treatment of colorectal cancer enables surgeons to successfully identify a higher number of synchronous abdominal neoplasms. Given the many technical advancements made in the field of laparoscopic surgery, synchronous, minimally invasive resections of coexisting abdominal disease have proven to be very feasible procedures. The laparoscopic resection of colorectal cancer paired with procedures like cholecystectomies for cholelithiasis has been recognized by the medical community as a reasonably practicable operation with no statistically significant increases in morbidity or mortality rates. On the other hand, synchronous laparoscopic resections for colorectal carcinoma and coexisting malignant cancers, (such as renal carcinoma) require superior technical expertise and lengthened operation and anesthesia times. Furthermore, the clinical advantages of synchronous laparoscopic surgery (LS) in the treatment of multiple malignancies have not yet been formally established from an oncological perspective.

Several randomized studies comparing LS and open surgery (OS) in the treatment of colorectal cancer have revealed many of the short-term advantages of LS, but the long-term oncological outcomes remain comparable for both techniques. Similarly, the long-term oncological results for LS and OS in the treatment of renal carcinoma are also comparable.

The authors recently encountered two cases of colorectal cancer and synchronous left and right renal carcinoma. Relatively early stage renal and colorectal cancer were discovered upon pre-operative examination, and subsequent synchronous LS procedures were performed successfully.

\section{Case Report 1}

A 68-year-old man was referred to the Division of General Surgery of the Ospedali Riuniti medical facilities for the treatment of early descending colon carcinoma, which had been diagnosed via colonoscopy following a positive fecal occult blood test performed during a previously scheduled regional screening program. The patient had no family history of Hereditary Nonpolyposis Colorectal Carcinoma (HNPCC). The colonoscopy showed a shallow ulcerated lesion, which, upon further examination, was found to be a T1 lesion that was non-resectable by means of endoscopy Biopsy results revealed high-grade displasia. Although 
pre-operative chest and abdominal CT scan showed no evidence of metastasis it revealed a right renal mass $(3.5 \mathrm{x}$ $3.2 \mathrm{~cm}$ ) with radiological features suggestive of neoplasm. Both malignancies were determined to be early stage carcinomas, and laparoscopic resection was performed two weeks after the initial consultation.

During surgery, five trocars were used (Fig. 1). Pneumoperitoneum was established using a Verres needle and a $12 \mathrm{~mm}$ Optic View (Ethicon Endo-Surgery, Germany) was introduced through a supraumbelical incision. Four other ports were then inserted by means of laparoscopic guidance.

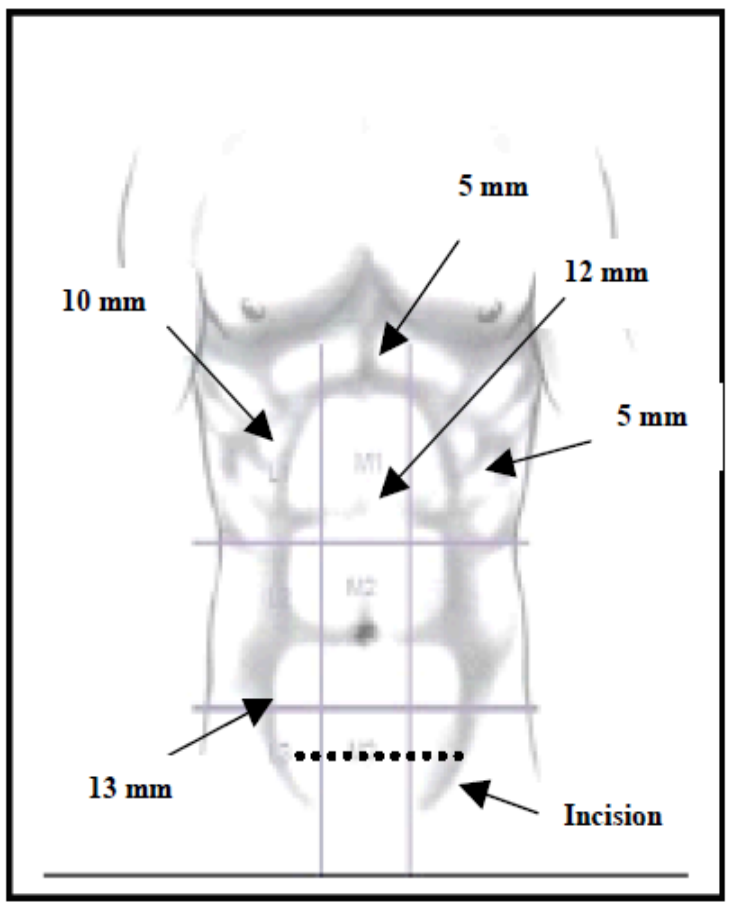

Figure 1. Trocar positioning for right nephrectomy and left colectomy

The patient was positioned on his left flank to allow for right kidney resection. The anterior face of the right kidney was exposed following complete mobilization of the right colon and duodenum. After identifying the right gonadal vessels and the right ureter on the right side of the aortic bifurcation, the right gonadal vessels were separated from each other. The right gonadal vessels and right ureter, together with the retroperitoneal adipose tissue, were dissected proximally, and the right gonadal artery was separated at the origin. The right gonadal vein, right renal vein, and right adrenal vein were then identified at the hilum of the kidney together with the right renal artery.

The right renal vein was separated using endoclips following separation of the right renal artery (Fig. 2). Removal of the right kidney with Gerota's fascia and adrenal gland was performed en bloc within an Endobag ${ }^{\circledR}$ (Ehticon Endo-Surgery, Germany) above the right side of the liver.

The patient was then moved to a supine and Trendelenburg position for the subsequent colectomy. After visualizing the inferior mesenteric artery and vein, an intracorporeal section was performed at the origin of the tumor's vascularization using a $10 \mathrm{~mm}$ LigaSure ${ }^{\circledR}$ (Covidien, Ireland) (Fig. 3). The left colon and sigmoid colon were mobilized using a medial-to-lateral approach in order to better identify and protect the left ureter and gonadal vessels. The lateral attachments of the sigmoid colon and left colon were then disjoined up until the splenic flexure, which had also been mobilized. The upper section of the rectum was mobilized, beginning with posterior mobilization along the avascular plain between the fascia propria of the rectum and the presacral fascia, followed by posterolateral dissection of the right and left regions of the rectum. The mesorectum and the rectosigmoid junction were partitioned from each other and the rectum was addressed using a $60 \mathrm{~mm}$ endoscopic linear stapler (Powered EndoGIA ${ }^{\circledR}$, Covidien, Ireland).

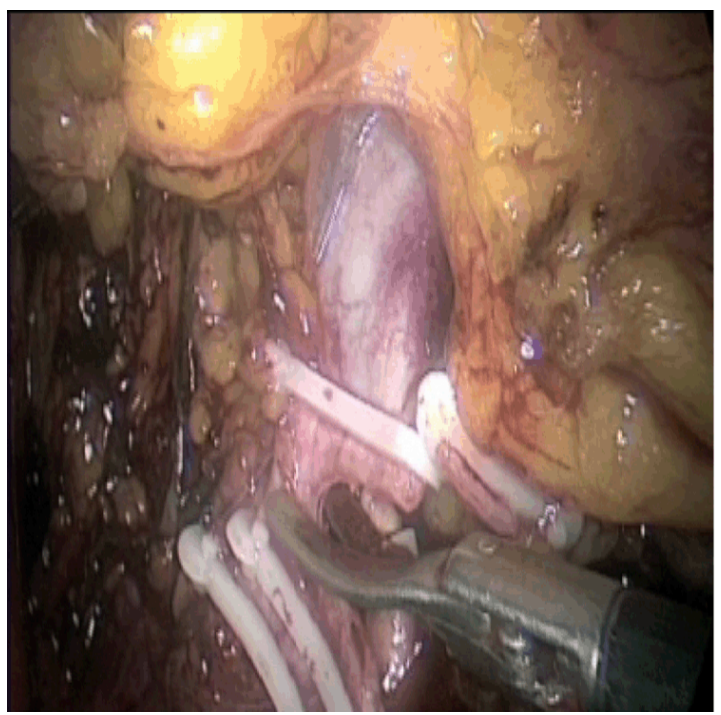

Figure 2. Right renal vessels section

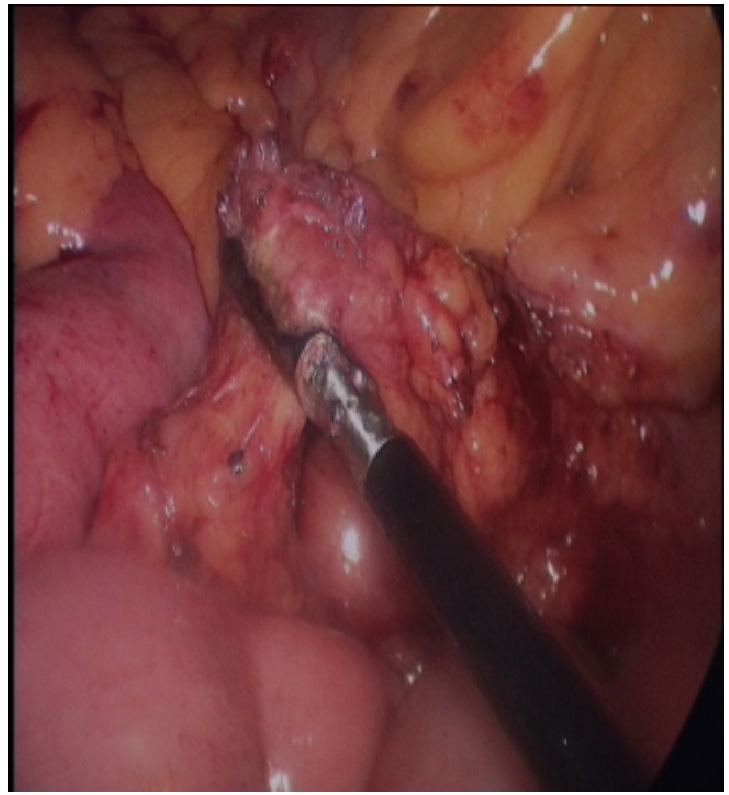

Figure 3. Inferior mesenteric vessels section

The right kidney was carefully extracted through a $10 \mathrm{~cm}$ 
Pfannestiel incision.

From the same incision, the left colon and sigmoid colon were then extracted to proceed with resection of the affected gastrointestinal loci. The envil of a $32 \mathrm{~mm}$ circular stapler (Ethicon Endo-Surgery, Germany) was then inserted into the proximal bowel. The bowel was carefully repositioned within the peritoneal cavity and the Pfannestiel laparotomy was subsequently closed. Pneumoperitoneum was re-established and an intracorporeal transanal anastomosis was performed with the stapler. Two abdominal drains were placed.

The total operation lasted 450 minutes with a total blood loss of $100 \mathrm{ml}$. The patient began eating liquid and solid foods post-operatively beginning on days 3 and 5, respectively. The patient was discharged on postoperative day 14, due to transient canalization disorders in post-operative day 9. Hystopathological examination of the colon confirmed the patient's pre-operative biopsy results (Adeno-carcinoma staging: pT1a G2 N0). Microscopic examination of the left kidney revealed a Fuhrman Grade 2 clear cell type carcinoma $(3.5 \mathrm{~cm})$. Ten months after the surgical procedure, the patient remains well and has shown no signs of recurrence or complication.

\section{Case Report 2}

A 70-year-old man was referred to the Division of Urology of the Ospedali Riuniti medical facilities for the treatment of renal cancer $(58 \times 63 \mathrm{~mm})$ of the left kidney. During pre-operative examination, a chest and abdomen CT scan showed a distal sigmoid neoplasm, which was later confirmed with a colonoscopy and biopsy analysis (Infiltrating adenocarcinoma). Both malignancies appeared to be early stage carcinomas, so simultaneous laparoscopic resections were then performed.

During surgery, five trocars were used (Fig. 4). Pneumoperitoneum was established using a Veress needle, and a $12 \mathrm{~mm}$ Optic View ${ }^{\circledR}$ (Ethicon Endo-Surgery, Germany) was introduced through a supraumbelical incision. Under laparoscopic oversight, two $5 \mathrm{~mm}$ ports, one $10 \mathrm{~mm}$ port, and one $13 \mathrm{~mm}$ port were then inserted.

The patient was positioned on his right flank to allow for resection of his left kidney. The patient was then adjusted to a supine and Trendelenburg position in order to perform the left hemicolectomy. The steps of the surgical procedure were the same as those outlined in the previous case.

The total operation lasted 380 minutes with a total blood loss of $150 \mathrm{ml}$. The patient began eating liquid and solid foods post-operatively beginning on days 3 and 5 , respectively. The post-operative recovery period was uneventful and the patient was discharged 8 days after surgery. The adenocarcinoma diagnosis was confirmed upon hystopathological examination of the colon (Staging: pT2 N1a M0). Microscopic examination of the right kidney revealed a Fuhrman Grade 2 clear cell type carcinoma $(6 \mathrm{~cm})$ (pT1b N0 M0). Eleven months after the surgical procedure, the patient remains well and has shown no signs of recurrence or complication.

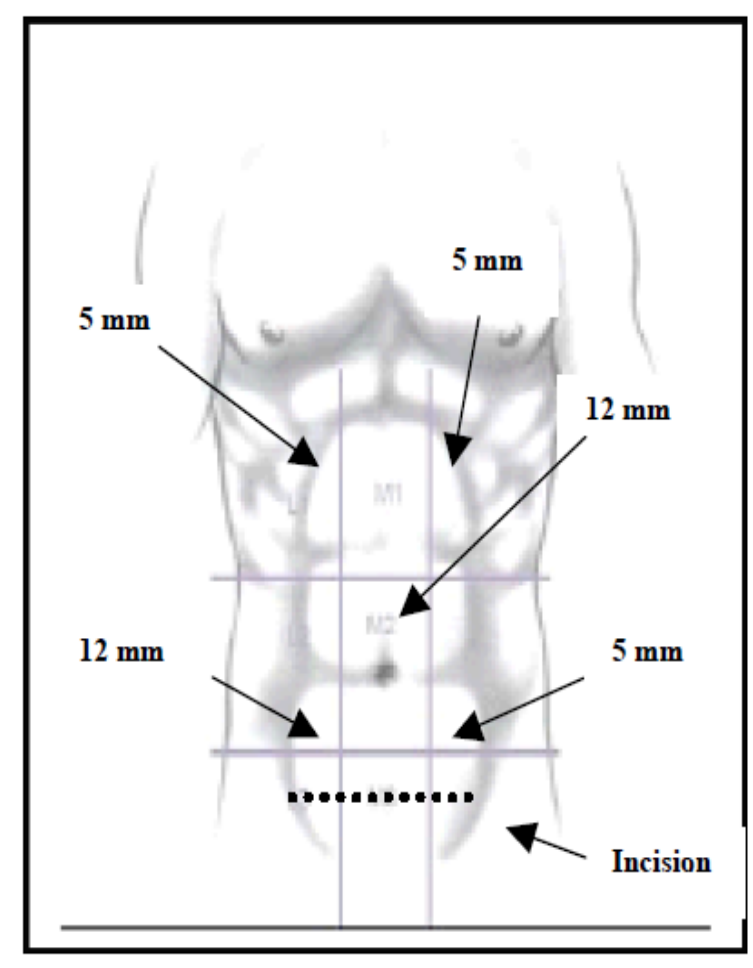

Figure 4. Trocar positioning for left nephrectomy and left colectomy

\section{Discussion}

Renal carcinoma has been reported in $0.03-4.8 \%$ of patients presenting with coexisting colorectal carcinoma (1, 2). Effective synchronous resections simultaneously addressing both colon and renal carcinomas by means of LS have been reported in several studies $(3,4,5,6,7)$.

Synchronous resections of both colorectal and renal neoplasms by means of conventional surgery, although technically feasible and more efficient than multiple back-to-back operations, are rife with formidable complications. For example, the separate incisions for the open resections are often incompatible with each other, leading to increased post-operative morbidity and mortality rates. Surgeons can often circumvent such problems by using the laparoscopic approach. During laparoscopic surgery, surgeons can reach different quadrants of the peritoneal cavity simultaneously by inserting extra-working ports. Additionally, the laparoscopic approach can offer improved visualization of the surgical field and the ability to effectively screen for conditions that may preclude resection (i.e., disseminated disease) (8). Compared to conventional open colorectal or urological surgery, the laparoscopic approach is associated with faster post-operative recovery periods and lower post-operative morbidity rates $(8,9,10)$, which can often allow for an earlier onset of adjuvant therapy. 
There are several issues that must be considered before performing synchronous laparoscopic resections to address coexisting abdominal neoplasms, including the intricate planning of port placement, patient positioning, and the assessment of which of the two resections should be performed first.

After collaborating with a series of urologists, the authors believe to have optimized the trocar placement in the aforementioned two cases. During both procedures, the surgeons did not experience any problems relating to the trocar placement, dissecting procedures, organ handling, or organ resection. Another issue of fundamental importance is patient positioning. By performing the nephrectomy first, the patient must only be repositioned once, moving from a lateral to supine position. In fact, immediately after the nephrectomy has been performed, the kidney is left above the liver inside the endobag, and the patient could easily be transitioned to a supine position to proceed with the colectomy and subsequent anastomosis.

Ultimately, the oncological outcome is the most important concern following such laparoscopic surgery. Ample evidence from mainstream literature suggests that laparoscopic resection of colorectal carcinoma is, at least from an oncological perspective, as safe as its open resection counterpart. $(4,10)$ Recent studies with intermediate and long-term data have also demonstrated that radical laparoscopic nephrectomies for localized renal malignancy have survival rates comparable to those of open surgery. (11, 12). That aside, many randomized and nonrandomized studies comparing LS and OS for colorectal or renal carcinomas have revealed that LS may exhibit several short-term advantages over OS. However, the overall long-term oncological outcomes achieved laparoscopically were recently found to be comparable to those achieved my means of open surgery $(11,13,14,15,16)$.

In conclusion, these reports have indicated that synchronous laparoscopic resection of colorectal and renal neoplasms is safe, effective, and technically feasible, and that it is associated with promising short- and long-term clinical and oncological outcomes.

\section{REFERENCES}

[1] Capra F, Scintu F, Zorcolo L, Marongiu L, Casula G. Synchronous colorectal and renal carcinomas. Is it a definite clinical entity? Chir Ital 2003;55:903-906.

[2] Halak M, Hazzan D, Kovacs Z, et al. Synchronous colorectal and renal carcinomas: a noteworthy clinical entity. Report of five cases. Dis Colon Rectum. 2000;43:1314-1315.

[3] Nishiyama N, Yamamoto S, Matsuoka N, Fujimoto H, Moriya Y. Simultaneous laparoscopic descending colectomy and nephroureterectomy for descending colon carcinoma and left ureteral carcinoma: report of a case Surg Today 2009;39:728-732

[4] Ng SSM, Lee JFY, Yiu RYC, Li JCM, Leung KL. Syncronous laparoscopic resection of colorectal and renal/adrenal neoplasm. Surg Laparosc Endosc Percutan Tech 2007; 17:283-286

[5] Ng SSM, Yiu RYC, Li JCM, Chan CK, Ng CF, Lau JYW Endolaparoscopic left hemicolectoomy and syncronous laparoscopic radical nephrectomy for obstructive carcinoma of the descending colon and renal cell carcinoma. J Laparoendosc Adv Surg Tech A 2006; 16: 297-300.

[6] Kim SH, Park JY, Joh YG, Hoe HE. Simultaneous laparoscopic radical nephrectomy and laparoscopic sigmoidectomy for renal cell carcinoma and colonic adenocarcinoma. J Laparoendosc Adv Surg Tech A 2004;14:179-181.

[7] Napolitano C Santoro G Valvano L Salvati V Martorano M. Simultaneous totally laparoscopic radical nephrectomy and laparoscopic left hemicolectomy for synchronous renal and sigmoid colon carcinoma: report of a case Int J Colorect Dis 2006;21: 92-93

[8] Cobb WS, Kercher KW, Sing RF, et al. Laparoscopic adrenalectomy for malignancy. Am J Surg 2005; 189:405-411.

[9] Raghuram S, Godbole HC, Dasgupta P. Laparoscopic nephrectomy: the new gold standard? Int J Clin Pract 2005;59:128-129.

[10] Leung KL, Kwok SP, Lam CW, et al. Laparoscopic resection of rectosigmoid carcinoma: a prospective randomised trial. Lancet. 2004;363:1187-1192.

[11] Permpongkosol S, Chan DY, Link RE, et al. Long-term survival analysis after laparoscopic radical nephrectomy. J Urol 2005; 174:1222-1225.

[12] Moinzadeh A, Gill IS. Laparoscopic radical adrenalectomy for malignancy in 31 patients. J Urol 2005;173:519-525.

[13] Lacy AM, García-Valdecasas JC, Delgado S, Castells A, Taurá P, Piqué JM, et al. Laparoscopy-assisted colectomy versus open colectomy for treatment of non-metastatic colon cancer: a randomised trial. Lancet 2002;359:2224-9.

[14] Fleshman J, Sargent DJ, Green E, Anvari M, Stryker SJ, Beart RW Jr, et al. Laparoscopic colectomy for cancer is not inferior to open surgery based on 5-year data from the COST Study Group trial. Ann Surg 2007;246:655-62.

[15] Jayne DG, Guillou PJ, Thorpe H, Quirke P, Copeland J, Smith AM, et al. Randomized trial of laparoscopic-assisted resection of colorectal carcinoma: 3-year results of the UK MRC CLASICC Trial Group. J Clin Oncol 2007;25:3061-8.

[16] Kahnamoui K, Cadeddu M, Farrokhyar F, Anvari M. Laparoscopic surgery for colon cancer: a systematic review. Can J Surg 2007;50:48-57. 